October 31 3 st, 2019

\title{
Found In Translation: a machine learning model for mouse-to-hu- man inference
}

This seminar was part of the Biolnfo4Women series.

\section{Rachelly Normand}

Shen-Orr lab at Technion - Israel Institute of Technology

\section{Abstract}

Cross-species differences form barriers to translational research that ultimately hinder the success of clinical trials, yet knowledge of species differences has yet to be systematically incorporated in the interpretation of animal models. We developed a machine learning model that leverages human and mouse public gene expression data to extrapolate the results of a new mouse experiment to expression changes in the equivalent human condition. We applied FIT to data from mouse models of 28 different human diseases and show it is able to identify $20-50 \%$ more human-relevant differentially expressed genes. FIT predicted novel disease-associated genes, an example of which we validated experimentally in Crohn's patients. FIT highlights signals that may otherwise be missed and reduces false leads with no experimental cost. It is available both as an $R$ package and as a web tool.

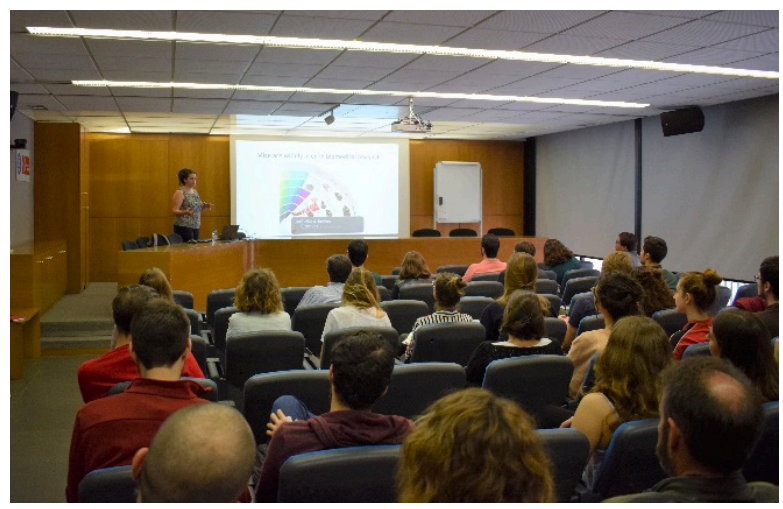




\section{Short bio}

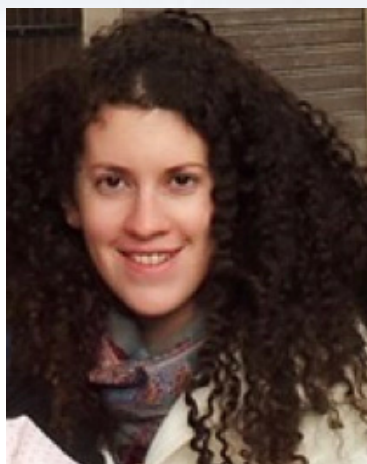

Rachelly Normand did her bachelor degree at the Computer Science Faculty at the Technion with emphasis in bioinformatics. She has worked for two years at the Technion Genome Center as a bioinformatician, analyzing high-throughput sequencing. She then did her master's and $\mathrm{PhD}$ in Prof. Shai Shen-Orr lab for systems immunology and precision medicine at the Medicine Faculty at the Technion. During her PhD Rachelly explored ways to improve translational research from mice to humans. She will soon start a post-doctoral fellowship at the Broad Institute and Harvard Medical School under the joint supervision of Prof. Aviv Regev and Prof. Chloe Villani and work on the Immune Cell Atlas Project. 